\title{
Full-field Strain Analysis of a Ski Boot
}

\author{
M. Reiter ${ }^{1}$, G. Singer ${ }^{2}$, and Z. Major ${ }^{1}$ \\ ${ }^{1}$ Institute of Polymer Product Engineering, Johannes Kepler University Linz; A-4040 Altenberger \\ Str. 69, Austria \\ ${ }^{2}$ Polymer Competence Center Leoben, A-8700 Leoben, Roseggerer Str 12, Austria
}

\begin{abstract}
The quality of the ski boots plays an extraordinary important role in the performance and in the safety of the skiers. The deformation behavior of a racing class ski boot was characterized by using the digital image correlation technique in this study. The boot was gripped in the ski binding and 3 types of motions of the skiers and the deformations of the boot were simulated by a professional skier in the laboratory. First, the buckles were closed in 4 stages and the resulting strains were measured. Furthermore, the skier positioned his balance continuously forward, resulting in a high overall bending deformation of the boot. The leg of the skier acted as a bending arm and pushed the upper part of the boot forward. This loading situation was assumed as quasistatic and was repeated several times. Finally, the skier jumped and this dynamic movement was recorded by using two high speed cameras for 3D analysis. Special focus was devoted to the measurement of the deformation of the boot during the contact of the ski with the ground of the laboratory. Both the displacement of the upper part and the local strain in selected areas of the boot was determined for both quasi-static and dynamic test conditions and are discussed in the paper.
\end{abstract}

\section{Introduction and Objectives}

Except of external influences like weather or snow for example, the following four basic factors are responsible for skiing performance: the ski, the binding, the boot and of course the skier himself. Skis can differ in their bending line, side cut, torsion-behaviour and attenuation. All of these parameters have an effect on the properties of a ski which can be felt by the skier. The quality of the ski boots plays also an extraordinary important role in the performance and in the safety of the skiers. While first is important for racing applications, later must be taken into account for the amateur sport [1]. Furthermore, there is a wide quality range for ski boots both from material and from design point of view. The ski boot is actually one of the most important elements in skiing. It is, together with the binding, the link between the skier and the ski. The ski boot is responsible to transport movements and forces initiated by the skier to steer the ski. If a boot is hard and stiff the load transmission is very fast and direct. That is good for advanced and racing skiers but a beginner needs a softer boot which tolerates mistakes and is less reactive. These requirements and diverse demands of the different skill levels lead to the development of various types of ski boots. One has to distinguish ski boots for beginners, intermediates and professionals and of course boots for children and for adults. The main difference lies in the material itself.

This is an Open Access article distributed under the terms of the Creative Commons Attribution-Noncommercial License 3.0, which permits unrestricted use, distribution, and reproduction in any noncommercial medium, provided the original work is properly cited. 
Professional racing boots significantly differ in their stiffness from a beginner boot, so the stiffness of a boot is one of its most important characteristics. On the other hand there is a difference in the construction of the boot. Most of the boots which are produced for adults have four buckles, while boots for children have three or even only one buckle. Boots for children are softer and are slower in their reaction to steering impulses because they are fitting more loosely. In modern ski boots a joint in the boot gives the flexibility to the ankle while the heel is fixed in its position. A well padded shaft and room to move the toes raise the comfort. A soft tongue prevents from pain on the shin. If the shaft is oriented into travel direction, forces are transmitted better to the ski and make the conversion into acceleration more efficient. Some boots allow adjusting the angle of leaning forward and the damping of the boot. The centre of gravity of the skier should be exact on the middle length axis of the ski, to minimize centrifugal forces. The axis of movement of the boot matches the natural axis of the ankle and guarantees more dynamic and powerful skiing. Canting, which means the sideways angle of the shaft can also be customized, to correct bowlegs or knock-knees [2]. The biggest influence on the performance of a ski boot has, apart from the material, the construction of the shell. In the history of development of ski boots not many different materials have been used. The first boots were made of leather, more or less directly followed by thermoplastic polyurethanes (TPUs) which are frequently used for recent ski boot models.

The objective of this paper is to characterize the deformation behaviour of a racing class ski boot and to determine the components of the local strains by using the digital image correlation technique. The results should support both material selection efforts and will be used for verifying further finite element simulations.

\section{Experimental}

The experiments were carried out using a high speed full-field digital image correlation system (Aramis, GOM, Braunschweig, D) and the set-up is shown in figure 1. The ski boot was prepared for the image correlation technique by applying a white and a black spray. While the black spray was used as the background the white spay produces a random distribution of spots in various sizes. This random distribution of the points results in a speckle muster and used for the stain analysis. The boot was gripped in the binding as it is shown in figure 2 . The local deformations and strains were measured in 3 test configurations. First, the strains were measured during the closing of the skiing boot by the clips (Test1). The closing of the clips results in local strains and represents a displacement controlled loading situation. In such loading situations polymeric materials reveal a time and temperature dependent relaxation. Furthermore, two significant type motions of the skiers were simulated by a professional skier acting as test person in the laboratory and this is shown in figure 3. The skier positioned his balance continuously forwards resulting in a high bending deformation of the boot. The leg of the skiers acted as a bending arm and pushed the upper part of the boot forward. This loading situation was assumed as quasi-static and was repeated by several times (Test2). Finally, during the third experiment a dynamic loading situation was simulated by the skier (Test3). The skier jumped and the movement was recorded by using high speed cameras with maximal frame rate $(2000 \mathrm{f} / \mathrm{s})$ and with sufficient image quality (512x256 pixels). Special focus was devoted to the measurement of the deformation of the boot during the contact of the ski with the ground of the laboratory. Both the temporal (stages) and the spatial (sections) distribution of the strains were measured. A representative image of the strain analysis is shown in figure 4 with section 1. 


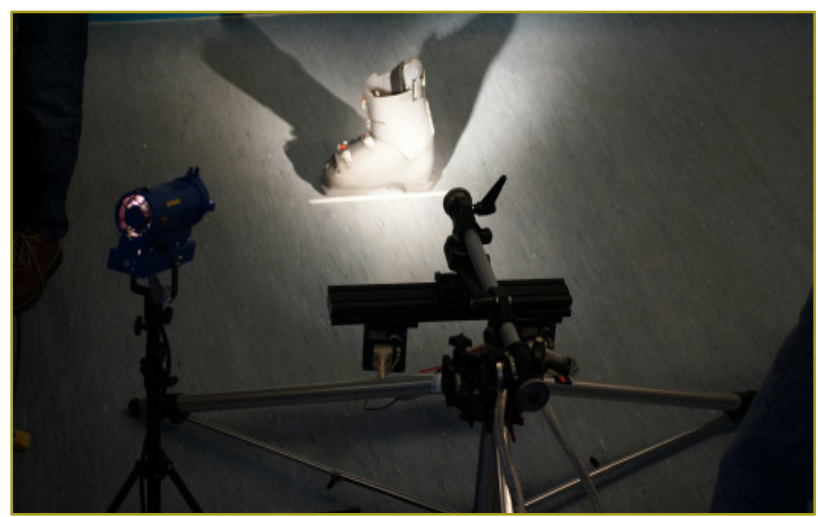

Fig. 1. The deformation of the boot was measured by a digital image correlation system (Aramis, GOM, Braunschweig, D).

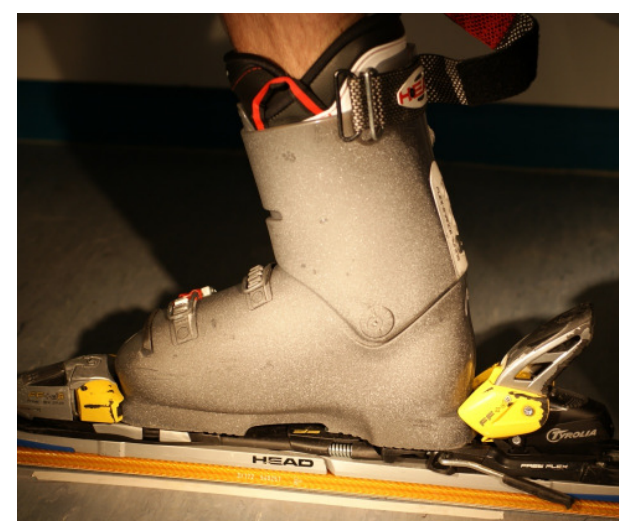

Fig. 2. The racing class skiing boot loaded by the leg of the skier within the binding and with the ski in the laboratory at $23^{\circ} \mathrm{C}$.

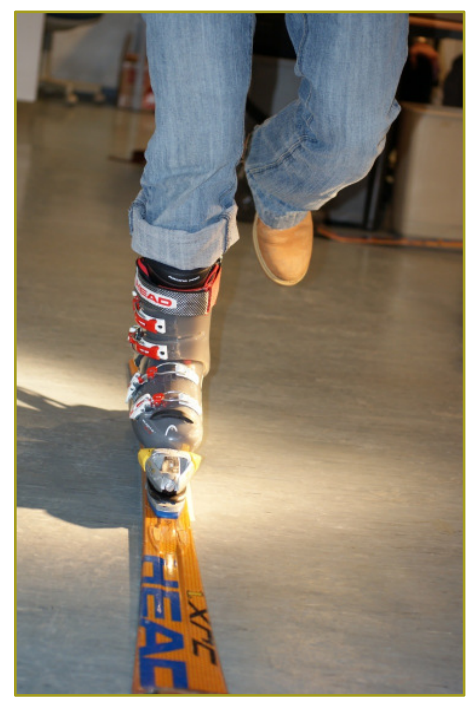

Fig. 3. The test person with the ski boot and the ski simulates the jumping movement. 


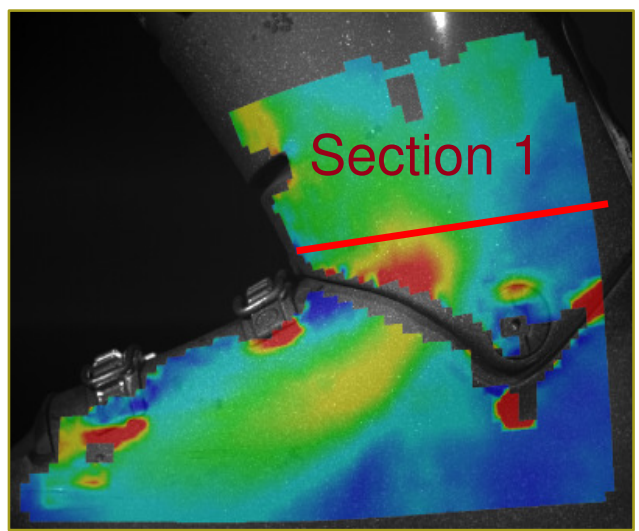

Fig. 4. Full-field strain analysis image of the ski boot.

\section{Results and Discussion}

To get more information about the initial strain situation a ski boot, first, the buckling process was analyzed. The 4 stages of the buckling are shown in figures $5 \mathrm{a}$ and $\mathrm{b}$. The spatial distribution of the strains was measured at all 4 stages. A linear increase of the major strain value with increasing section length and a maximal strain value of $2.5 \%$ was observed after the fourth stages.

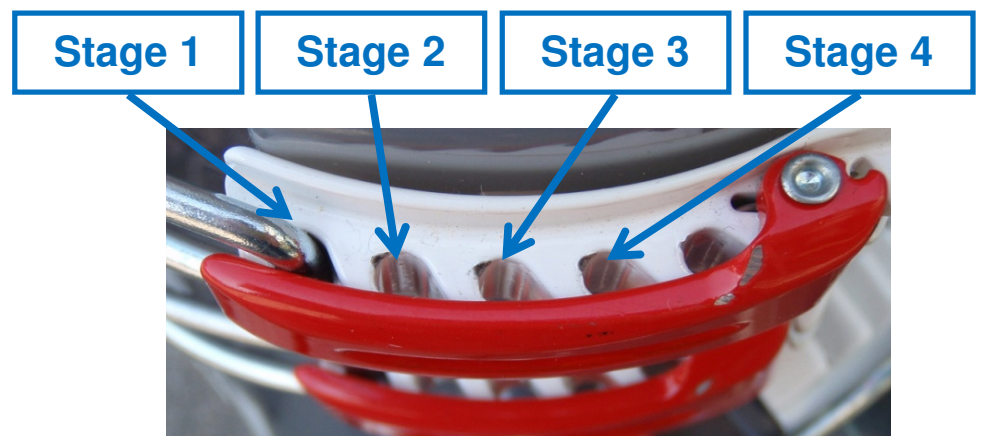

(a)

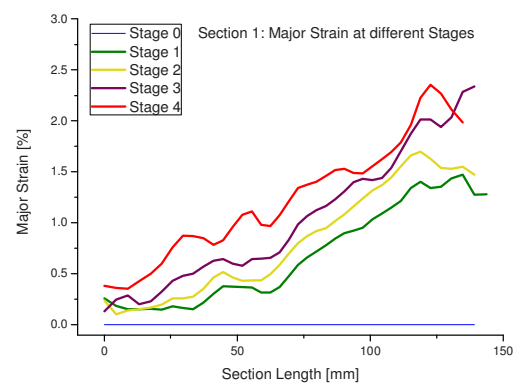

(b)

Fig. 5. The 4 stages of the buckling; (a) the buckle and (b) the major strain in section 1 during the buckling process. 
Furthermore, after finishing the buckling, the skier positioned his balance continuously forwards resulting in a high bending deformation of the boot (Test 2). The leg of the skiers acted as a bending arm and pushed the upper part of the boot forward. This loading situation was assumed as quasi-static and was repeated by several times. The major strain, $\varepsilon_{\mathrm{M}}$ distribution of both sides of the skiing boot during this type of loading is shown in figure 6. The small black arrows indicate the direction of the major strain. The forward lean angle dependence of the maximal value of the major strain for these tests is shown in figure 7. Similar to the previous test (Test 1) a near linear increase of the maximal value of the major strain with increasing forward lean angle (up to $8^{\circ}$ ) up to about $5.5 \%$ strain was observed.

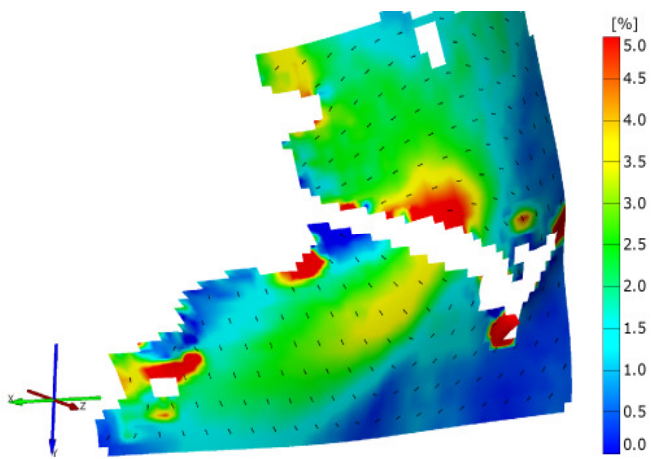

(a)

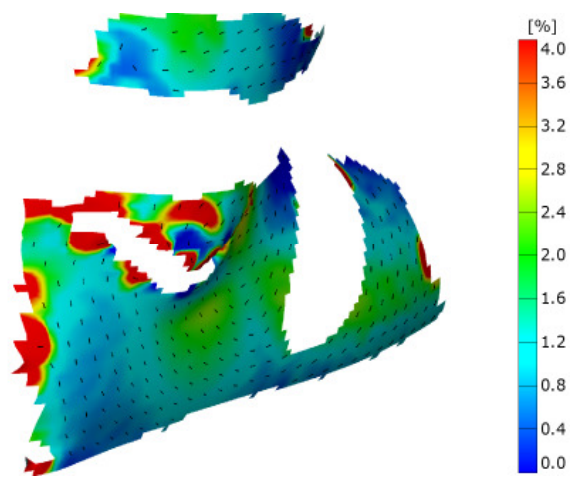

(b)

Fig. 6. Strain analysis of the skiing boot; (a) inner side and (b) outer side with the clips. The major strain, $\varepsilon_{M}$ directions are depicted in the figure.

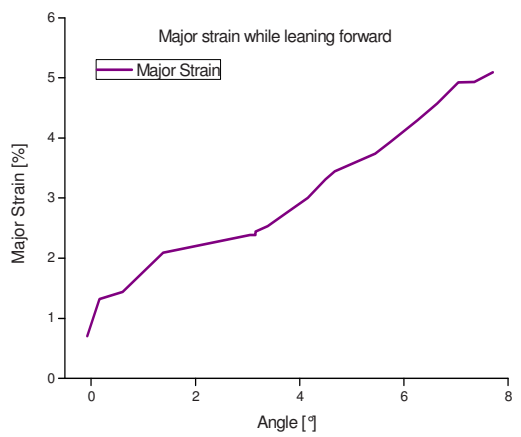

Fig. 7. The forward lean angle dependence of the maximal value of the major strain for Test 2.

The localization of the strain in various positions of the ski boot is also visible in figure 6 . These images provide a good basis for changing the construction and make the boot stiffer.

Finally, during the third experiment (Test 3) a dynamic loading situation was simulated by the skier. In spite of the fact that this low rate jumping is far from the dynamic loading situation of the boot during the real skiing process, the test yielded valuable information about the time dependent nature of the deformations process. Both the displacement of the upper part and the local strain in selected areas of the boot was determined for dynamic test conditions. Furthermore, 3 positions were selected on the boot, assigned in the 3D image and the time history of a jump (temporal distribution of the strains) was determined and it is shown in figure 8. 

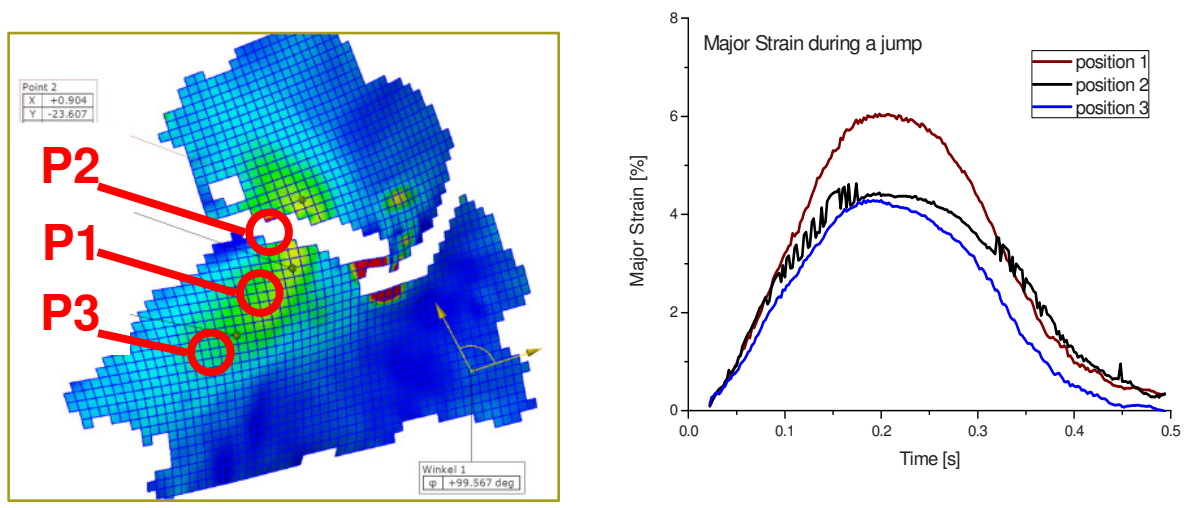

Fig. 8. The temporal distribution of the major strain in 3 locations selected on the boot surface for test 3 .

The maximal value of the major strain was observed somewhat higher than in Test 2 and was at about $6-6.5 \%$. While the first increasing part of the strain vs. time curve (up to the maximum value) is relevant for the dynamic loading process, the decreasing part shows the subsequent strain relaxation. It must be mentioned, however, that the contact stiffness (the quality of the snow) may significantly affect the shape of the curve and the behaviour of the ski boot. Nevertheless, the character of the process remains similar and reveals the importance of a proper dynamic mechanical analysis for material selection.

\section{Conclusions and Outlook}

Full-field deformation and strain analysis of a racing class ski boot was performed in this study. While strain measurement and the data reduction of the quasi-static experiments /Test 1 and Test 2) were relatively easy, the dynamic experiments (Test 3) were more complicated. Relatively high strains up to about $6.5 \%$ were measured in the ski boot at various positions during these experiments. Test 1 provided valuable information about the initial state of strain, Test 2 about the spatial distribution of the strains and strain localization during the loading and Test 3 provided the temporal distribution of the strain during a dynamic loading process. Based on above experiments the relevant strain range for subsequent material testing was determined. Time/rate and temperature dependent material parameters for modeling were determined in this strain range and these results will be reported in a following paper.

\section{References}

1. Singer, G., 2010, B .Sc Thesis, University of Leoben

2. Snowsports Academy Austria, May 2004

\section{Acknowledgments}

Parts of this project were performed at the Polymer Competence Center Leoben GmbH within the $\mathbf{K}_{\text {plus }}{ }^{-}$ programme of the Austrian Ministry of Traffic, Innovation and Technology. The funding within this programme by the Governments of Austria, Styria and Upper Austria is gratefully acknowledged. The racing class ski boot was provided for these experiments by Mr. H. Schretter Dir. R\&D HEAD TYROLIA Wintersport and a special thanks goes to him for the valuable discussions. 